\title{
Isolation and Quantitative Analysis of BACE1 Inhibitory Compounds from Cirsium maackii Flower
}

\author{
Grishma Bhatarrai ${ }^{1}$, Su Hui Seong ${ }^{1}$, Hyun Ah Jung ${ }^{2, *}$, and Jae Sue Choi ${ }^{1, *}$ \\ ${ }^{1}$ Department of Food and Life Sciences, Pukyoung National University, Busan 48513, Republic of Korea \\ ${ }^{2}$ Department of Food Science and Human Nutrition, Jeonbuk National University, Jeonju 54896, Republic of Korea
}

\begin{abstract}
The purpose of our study was to evaluate anti-AD potential of Cirsium maackii flowers. MeOH extract, $\mathrm{CH}_{2} \mathrm{Cl}_{2}$, EtOAc, and $n$-BuOH fraction of this flower notably inhibited BACE1 $\left(\mathrm{IC}_{50}=76.47 \pm 1.66\right.$, $22.98 \pm 1.45,8.65 \pm 0.63$, and $72.47 \pm 3.04 \mu \mathrm{g} / \mathrm{mL}$, respectively). $\beta$-amyrenone (49.70 $\mathrm{mg})(\mathbf{1})$, lupeol acetate (1.43 g) (2), lupeol (1.22 g) (3), lupenone (23.70 mg) (4), $\beta$-sitosterol (1.01 g) (6), and $\beta$-sitosterol glucoside (13.00 mg) (7) from $\mathrm{CH}_{2} \mathrm{Cl}_{2}$, apigenin (100.20 mg) (8), luteolin (19.00 mg) (9), apigenin 7-O-glucuronide methyl ester $(21.30 \mathrm{mg})(\mathbf{1 4})$, and tracheloside $(53.70 \mathrm{mg})(\mathbf{5})$ from EtOAc, apigenin 5-O-glucoside (11.00 $\mathrm{mg})(\mathbf{1 0})$, luteolin 5-O-glucoside $(11.00 \mathrm{mg})(\mathbf{1 1})$ and apigenin $7-O$-glucuronide $(91.00 \mathrm{mg}$ ) (12) from $n$ - $\mathrm{BuOH}$, and luteolin 7-O-glucuronide $(22.00 \mathrm{mg})(\mathbf{1 3})$ from $\mathrm{H}_{2} \mathrm{O}$ fraction were isolated. HPLC showed high levels of $\mathbf{8 ,}, \mathbf{9}$ and 12 in $\mathrm{MeOH}$ extract $(33.07 \pm 0.07,31.44 \pm 0.17$ and $16.89 \pm 0.33 \mathrm{mg} / \mathrm{g}$, respectively), EtOAc $(161.01 \pm 1.78$, $96.93 \pm 0.34$ and $73.38 \pm 0.06 \mathrm{mg} / \mathrm{g}$, respectively), and $n$-BuOH fraction $(32.18 \pm 0.33,44.31 \pm 0.32$ and 105.94 $\pm 0.36 \mathrm{mg} / \mathrm{g}$, respectively). Since, 3 and $\mathbf{9}$ are well-known BACE1 inhibitors, the anti-AD activity of C. maackii flower might be attributable to their presence.
\end{abstract}

Keywords - Cirsium maackii flower, Alzheimer's disease, BACE1, HPLC, Isolation

\section{Introduction}

Alzheimer's disease (AD) is a progressive neurodegenerative disease where brain regions, particularly cerebral cortex and hippocampus, are affected by deposition of amyloid- $\beta$ (A $\beta$ ) plaques and neurofibrillary tangles accompanied by synaptic dysfunction and neurodegeneration. ${ }^{1,2}$ Increased proteolytic processing of $\beta$-amyloid precursor protein (APP) can lead to the production and accumulation of neurotoxic forms of $\mathrm{A} \beta$ in the brain. ${ }^{3}$ According to the amyloid cascade hypothesis, APP is generally cleaved by $\alpha$-secretase and simultaneously processed by $\beta$ - and $\gamma$-secretases. Disparity between production and clearance of $A \beta$ peptide can result in aggregation of $A \beta$ peptide into soluble oligomers and forms insoluble fibrils of beta-sheet conformation that are eventually deposited in diffused senile plaques. ${ }^{4}$ Betasecretase 1, also known as beta-site amyloid precursor

\footnotetext{
*Author for correspondence

Hyun Ah Jung, Department of Food Science and Human Nutrition, Jeonbuk National University, Jeonju 54896, Republic of Korea. Tel: +82-63-270-4882; E-mail: jungha@jbnu.ac.kr

Jae Sue Choi, Department of Food and Life Sciences, Pukyoung National University, Busan 48513, Republic of Korea.

Tel: +51-629-5845; E-mail: choijs@pknu.ac.kr
}

protein cleaving enzyme 1 (BACE1) is an enzyme that leads to the production of neurotoxic $A \beta$ in the pathogenesis of $\mathrm{AD}$. Due to the result disabling nature and chronicity of $\mathrm{AD}$ with high prevalence in older population and inability of available $\mathrm{AD}$ treatments to justify their costs, current $\mathrm{AD}$ treatments have been criticized because they fail to provide sufficient benefit.

Emerging researches have been directed toward BACE1 inhibitors due to their potential in lowering cerebral $A \beta$ concentrations and treating as well as preventing $\mathrm{AD} .^{5}$ Moreover, BACE1 initiates the production of toxic $A \beta$ that plays a crucial role in the early phase of $\mathrm{AD}$ pathogenesis. Due to its apparent rate limiting function, BACE1 appears to be a prime target to prevent and lower $\mathrm{A} \beta$ generation in $\mathrm{AD} .^{6}$

Cirsium maackii (C. maackii) is an herbaceous perennial plant of family Compositae. It is native to Korea. ${ }^{7}$ Roots or whole plant of Cirsii Radix et Herba have been used as a folk medicine to treat hemorrhage, diuresis, inflammation of the liver and kidney, and a variety of abdominal and intestinal disorders. ${ }^{8}$ Cirsium species have been found to exert various bioactivities, including anti-microbial, ${ }^{9}$ anti-proliferative, ${ }^{10}$ anti-diabetic, ${ }^{11}$ anti-oxidant, ${ }^{12}$ anti-inflammatory, ${ }^{7}$ vasorelaxant, ${ }^{13}$ anti-cancer, ${ }^{14}$ and hepatopro- 
tective ${ }^{15}$ effects. In addition, numerous phytochemicals, including flavonoids, phenolic acids, ${ }^{9}, 16$ lignans, neolignans, sesquineolignans, ${ }^{16,17}$ phenylacrylic acid esters, polyacetylenes, ${ }^{18}$ sterols, and triterpenes have been isolated from these species. ${ }^{19,20}$

Walesiuk et al. have reported that Cirsium rivulare flowers extract shows pro-cognitive and its leaf extract possesses anxiolytic effect in a rat model. ${ }^{21} \mathrm{Kim}$ and Kim have shown that, among methanol extracts of root, stem, leaves, and flowers of Cirsium japonicum, flowers and leaves have optimum peaks of luteolin, apigenin, and hispidulin when analyzed by high-performance liquid chromatography (HPLC). ${ }^{22}$

Our previous study has focused on inhibitory effects of C. maackii leaves on advanced glycation end products (AGEs). ${ }^{16}$ Considering mounting interest in assessing the anti-AD capacity of compounds from natural source and the ease of access to Cirsium species, the objective of the present study was to determine whether $C$. maackii flower could alleviate $\mathrm{AD}$ by evaluating its BACE1 inhibition effect and clarifying its activity through quantitative analysis of compounds present in its different extracts and fractions by HPLC.

\section{Experimental}

Chemicals and reagents - BACE1 kit was obtained from PanVera Corp. (Madison, WI, USA). Ultra-pure grade water was used in this study. All chemicals and solvents used in this study were purchased from SigmaAldrich (St. Louis, MO, USA) unless otherwise stated. All solvents used in HPLC were of HPLC grade.

Plant materials-Flowers of $C$. maackii were collected from the southeast area of Gyeongsangnam-do Province, Korea, in 2016 and authenticated by Professor Jae Sue Choi (Pukyong national university, Korea). A voucher specimen (No.20160901) was deposited at the laboratory of Professor J.S. Choi.

Extraction, fractionation, and isolation - Dried flowers of C. maackii $(2.8 \mathrm{~kg})$ were extracted four times with $\mathrm{MeOH}$ at $70^{\circ} \mathrm{C}$. The final yield of $\mathrm{MeOH}$ extract was $168.28 \mathrm{~g}$. The obtained extract was then suspended in water $\left(\mathrm{H}_{2} \mathrm{O}\right)$ and successively partitioned with dichloromethane $\left(\mathrm{CH}_{2} \mathrm{Cl}_{2}\right)$, ethyl acetate (EtOAc), and $n$-butanol $(n-\mathrm{BuOH})$ to yield $\mathrm{CH}_{2} \mathrm{Cl}_{2}(34.78 \mathrm{~g})$, EtOAc $(13.33 \mathrm{~g})$, and $n$ - $\mathrm{BuOH}(16.63 \mathrm{~g})$ fractions, as well as $\mathrm{H}_{2} \mathrm{O}$ residue $(102.00 \mathrm{~g})$. The $\mathrm{CH}_{2} \mathrm{Cl}_{2}$ fraction was chromatographed over silica gel column and eluted with $n$-hexane:EtOAc 25:1 to EtOAc to obtain five subfractions. Repeated chromatography of subfraction 1 yielded $\beta$-amyrenone
$(49.70 \mathrm{mg})(\mathbf{1})$, lupeol acetate (1.43 g) (2), and lupenone $(23.70 \mathrm{mg})(4)$ while subfraction 2 yielded lupeol $(1.22 \mathrm{~g})$ (3). $\beta$-sitosterol $(1.01 \mathrm{~g})(6)$ and $\beta$-sitosterol glucoside $(13.00 \mathrm{~g})(7)$ were obtained from subfractions 3 and 5 , respectively. The EtOAc fraction was subjected to silica gel column chromatography and eluted with $\mathrm{CH}_{2} \mathrm{Cl}_{2}$ : $\mathrm{MeOH}: \mathrm{H}_{2} \mathrm{O}$ (7:1:0.1 to 3:1:0.1) to give seven different subfractions. Apigenin $(100.20 \mathrm{mg})(\mathbf{8})$ was obtained as a precipitate from subfraction 2 . Repetitive chromatography of subfractions 3, 4, and 5 over silica gel column with $\mathrm{CH}_{2} \mathrm{Cl}_{2}: \mathrm{MeOH}: \mathrm{H}_{2} \mathrm{O}$ (10:2:0.1, 7:1:0.1, and 5:1:0.1) yielded luteolin $(19.00 \mathrm{mg})(\mathbf{9})$, tracheloside $(53.70 \mathrm{mg})(\mathbf{5})$, and apigenin 7-O-glucuronide methyl ester $(21.30 \mathrm{mg})(\mathbf{1 4})$, respectively.

The $n$ - $\mathrm{BuOH}$ fraction was proceeded to chromatography on Sephadex LH-20 column using $100 \% \mathrm{MeOH}$ as a mobile phase solvent to yield eight different subfractions. Continual elution of subfraction 4 using EtOAc: $\mathrm{MeOH}: \mathrm{H}_{2} \mathrm{O}$ (24:4:3) and $\mathrm{RP}_{-} \mathrm{C}_{18}$ column gave apigenin 7-O-glucuronide $(91.00 \mathrm{mg})(\mathbf{1 2})$ whereas subfraction 7 chromatographed over silica gel column using EtOAc: $\mathrm{MeOH}: \mathrm{H}_{2} \mathrm{O}$ (30:2:1) gave an insoluble precipitate on addition of $\mathrm{MeOH}$. This precipitate was separated through filtration and later identified as luteolin 5-O-glucoside (11.00 mg) (11). Repetitive column chromatography of the supernatant over silica gel, RP-C $\mathrm{C}_{18}$, and Sephadex LH20 yielded apigenin 5-O-glucoside (11.00 mg) (10). Quarter amount of the $\mathrm{H}_{2} \mathrm{O}$ fraction $(40 \mathrm{~g})$ was subjected to Diaion HP-20 column and eluted with $100 \% \mathrm{H}_{2} \mathrm{O}$ and $100 \% \mathrm{MeOH}$. Continuous silica gel, Sephadex LH-20, and MCI CHP-20P chromatography of $100 \% \mathrm{MeOH}$ subfraction (7 g) with EtOAc:MeOH: $\mathrm{H}_{2} \mathrm{O}(24: 4: 3), 50 \%$ $\mathrm{MeOH}$, and $30 \% \mathrm{MeOH}$ gave luteolin 7-O-glucuronide (22.00 mg) (13).

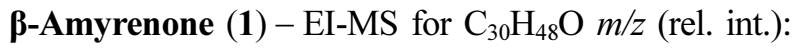
$409\left[\mathrm{M}^{-\mathrm{CH}_{3}}\right](4.59 \%), 218.25$ (100\%), 203.15 (34\%), 189.20 (24.21\%), 147.15 (11.88\%).

Lupeol acetate (2)-EI-MS for $\mathrm{C}_{32} \mathrm{H}_{52} \mathrm{O}_{2} \mathrm{~m} / z$ (rel. int.): $468\left[\mathrm{M}^{+}\right](20.99 \%), 408.35\left[\mathrm{M}-\mathrm{CH}_{3} \mathrm{COO}\right]^{+}(8.36 \%)$, $189.15(100 \%)$.

Tracheloside (5) $-{ }^{1} \mathrm{H}-\mathrm{NMR}\left(\mathrm{CD}_{3} \mathrm{OD}, 600 \mathrm{MHz}\right) \delta_{\mathrm{H}}$ $7.06\left(1 \mathrm{H}, \mathrm{d}, J=8.3 \mathrm{~Hz}, \mathrm{H}-5^{\prime}\right), 6.85(1 \mathrm{H}, \mathrm{d}, J=7.6 \mathrm{~Hz}, \mathrm{H}-$ 5), $6.70(1 \mathrm{H}, \mathrm{d}, J=2.1 \mathrm{~Hz}, \mathrm{H}-2), 6.68(3 \mathrm{H}, \mathrm{d}, \mathrm{H}-6), 6.67$ $\left(2 \mathrm{H}, \mathrm{d}, J=2.1 \mathrm{~Hz}, \mathrm{H}-2^{\prime} / 6^{\prime}\right), 3.98$ (2H, d, $\left.J=8.9 \mathrm{~Hz}, \mathrm{H}-9\right)$, $3.14\left(1 \mathrm{H}, \mathrm{d}, J=13.7 \mathrm{~Hz}, \mathrm{H}-7^{\prime}\right), 2.88(1 \mathrm{H}, \mathrm{d}, J=13.7 \mathrm{~Hz}$, H-7'), $2.79(1 \mathrm{H}, \mathrm{dd}, J=13.7,5.52 \mathrm{~Hz}, \mathrm{H}-7), 2.51(1 \mathrm{H}, \mathrm{dd}$, $J=13.8,9.63 \mathrm{~Hz}, \mathrm{H}-7), 2.43(1 \mathrm{H}, \mathrm{m}) .{ }^{13} \mathrm{C}-\mathrm{NMR}\left(\mathrm{CD}_{3} \mathrm{OD}\right.$, $150 \mathrm{MHz}) \delta_{\mathrm{c}} 180.37$ (C-9'), $150.61\left(\mathrm{C}-4^{\prime}\right), 150.58(\mathrm{C}-4)$, 149.18 (C-3), 147.17 (C-3'), 133.34 (C-1), 131.71 (C-1'), 124.13 (C-6'), 122.23 (C-6), 117.82 (C-5'), 115.94 (C-2'), 
113.96 (C-2), 113.25 (C-5), 102.85 (C-1"), 77.27 (C-8'), 77.84 (C- 3"), 74.89 (C-2"), 71.78 (C-9), 71.30 (C-4"), 44.60 (C-8), 41.83 (C-7'), 32.18 (C-7).

Apigenin 7-O-glucuronide methyl ester $(14)-{ }^{1} \mathrm{H}$ NMR (DMSO- $\left.d_{6}, 600 \mathrm{MHz}\right) \delta_{\mathrm{H}} 7.95(2 \mathrm{H}, \mathrm{d}, J=8.9 \mathrm{~Hz}$, H-2'/6'), 6.94 (2H, d, $\left.J=8.8 \mathrm{~Hz}, \mathrm{H}-3^{\prime} / 5^{\prime}\right), 6.86(1 \mathrm{H}, \mathrm{s}, \mathrm{H}-$ 3), $6.85(1 \mathrm{H}, \mathrm{d}, J=2.0 \mathrm{~Hz}, \mathrm{H}-8), 6.47(1 \mathrm{H}, \mathrm{d}, J=2.7 \mathrm{~Hz}$, H-6), $5.32\left(2 \mathrm{H}, \mathrm{d}, J=7.6 \mathrm{~Hz}, \mathrm{H}-1^{\prime \prime}\right), 4.21(1 \mathrm{H}, \mathrm{d}, J=9.6$ $\left.\mathrm{Hz}, \mathrm{H}-5^{\prime}\right), 3.67\left(3 \mathrm{H}, \mathrm{s}, \mathrm{OCH}_{3}\right) .{ }^{13} \mathrm{C}-\mathrm{NMR}$ (DMSO- $d_{6}, 150$ MHz) $\delta_{\mathrm{c}} 181.95$ (C-4), 169.16 (C-6'), 164.30 (C-2), 162.39 (C-7), 161.46 (C-4'), 161.16 (C-5), 156.93 (C-9), 128.59 $\left(\mathrm{C}-2^{\prime} / 6^{\prime}\right), 120.90\left(\mathrm{C}-1^{\prime}\right), 116.00\left(\mathrm{C}-3^{\prime} / 5^{\prime}\right), 105.96(\mathrm{C}-10)$, 103.08 (C-3), 99.28 (C-6), 94.60 (C-8), 75.38 (C-5'), $75.13\left(\mathrm{C}-2^{\prime}\right), 72.69$ (C-3'), $71.28\left(\mathrm{C}-4^{\prime}\right), 51.95\left(\mathrm{OCH}_{3}\right)$.

Apigenin 5-O-glucoside (10) $-{ }^{1} \mathrm{H}$ NMR (DMSO- $d_{6}$, $600 \mathrm{MHz}) \delta_{\mathrm{H}} 7.88\left(1 \mathrm{H}, \mathrm{d}, J=9.0,2.2 \mathrm{~Hz}, \mathrm{H}-2^{\prime} / 6^{\prime}\right), 6.91$ $\left(1 \mathrm{H}, \mathrm{d}, J=8.9 \mathrm{~Hz}, \mathrm{H}-5^{\prime}\right), 6.79(1 \mathrm{H}, \mathrm{d}, J=2.0 \mathrm{~Hz}, \mathrm{H}-8)$, $6.65(1 \mathrm{H}, \mathrm{s}, \mathrm{H}-3), 6.73(1 \mathrm{H}, \mathrm{d}, J=2.5 \mathrm{~Hz}, \mathrm{H}-6), 4.70(1 \mathrm{H}$, d, $J=7.7 \mathrm{~Hz}, \mathrm{H}-1{ }^{\prime \prime}$ ), ${ }^{13} \mathrm{C}$ NMR (DMSO- $\left.d_{6}, 150 \mathrm{MHz}\right) \delta_{\mathrm{C}}$ 176.99 (C-4), 162.69 (C-2), 161.18 (C-7), 158.64 (C-9), 158.32 (C-5), 160.76 (C-4'), $115.87\left(\mathrm{C}-3^{\prime} / 5^{\prime}\right), 128.08$ (C2'/6'), 121.16 (C-1'), 108.17 (C-10), 104.56 (C-3), 104.40 (C-6), 98.30 (C-8), 75.57 (C-3"), 77.57 (C-5"), 73.62 (C2"), 69.66 (C-4"), 60.82 (C-6").

Apigenin 7-O $\boldsymbol{O}$-glucuronide (12) - ${ }^{1} \mathrm{H}$ NMR (DMSO$\left.d_{6}, 400 \mathrm{MHz}\right) \delta_{\mathrm{H}} 7.85\left(2 \mathrm{H}, \mathrm{d}, J=8.9 \mathrm{~Hz}, \mathrm{H}-2^{\prime} / 6^{\prime}\right), 6.88$ ( $\left.2 \mathrm{H}, \mathrm{d}, J=8.9 \mathrm{~Hz}, \mathrm{H}-3^{\prime} / 5^{\prime}\right), 6.77(1 \mathrm{H}, \mathrm{d}, J=4.2 \mathrm{~Hz}, \mathrm{H}-8)$, $6.40(1 \mathrm{H}, \mathrm{s}, \mathrm{H}-3), 6.40(1 \mathrm{H}, \mathrm{d}, J=2.0 \mathrm{~Hz}, \mathrm{H}-6), 5.05(1 \mathrm{H}$, $\left.\mathrm{d}, J=6.6 \mathrm{~Hz}, \mathrm{H}-1^{\prime \prime}\right) .{ }^{13} \mathrm{C}$ NMR (DMSO- $\left.d_{6}, 100 \mathrm{MHz}\right) \delta_{\mathrm{C}}$ 181.92 (C-4), 172.45 (C-6"), 164.41 (C-2), 163.03 (C-7), 156.94 (C-9), 162.51 (C-5), 161.0 (C-4'), 116.16 (C-3'/5'), $128.44\left(\mathrm{C}-2^{\prime} / 6^{\prime}\right), 120.16\left(\mathrm{C}-1^{\prime}\right), 105.26$ (C-10), 102.64 (C3), 99.61 (C-6), 94.65 (C-8), 73.75 (C-3"), 76.48 (C-5"), 72.95 (C-2"), 71.98 (C-4").

Luteolin 7-O-glucuronide (13) $-{ }^{1} \mathrm{H}$ NMR (DMSO- $d_{6}$, $600 \mathrm{MHz}) \delta_{\mathrm{H}} 7.42\left(1 \mathrm{H}, \mathrm{s}, \mathrm{H}-2^{\prime}\right), 7.38(1 \mathrm{H}, \mathrm{d}, J=8.7 \mathrm{~Hz}$, H-6'), $6.86\left(1 \mathrm{H}, \mathrm{d}, J=7.9 \mathrm{~Hz}, \mathrm{H}-5^{\prime}\right), 6.76(1 \mathrm{H}, \mathrm{d}, J=1.4$ $\mathrm{Hz}, \mathrm{H}-8), 6.69(1 \mathrm{H}, \mathrm{s}, \mathrm{H}-3), 6.40(1 \mathrm{H}, \mathrm{d}, J=2.1 \mathrm{~Hz}, \mathrm{H}-6)$, $5.09\left(1 \mathrm{H}, \mathrm{d}, J=7.6 \mathrm{~Hz}, \mathrm{H}-1^{\prime \prime}\right) .{ }^{13} \mathrm{C}$ NMR (DMSO- $d_{6}, 150$ MHz) $\delta_{\mathrm{C}} 181.80(\mathrm{C}-4), 172.70\left(\mathrm{C}-6^{\prime \prime}\right), 162.95$ (C-2), 164.46 (C-7), 160.98 (C-5), 156.89 (C-9), 150.34 (C-4'), $145.96\left(\mathrm{C}-3^{\prime}\right), 118.96$ (C-1'), 120.90 (C-6'), 116.04 (C-5'), 113.11 (C-2'), 105.23 (C-10), 102.83 (C-3), 99.54 (C-1"), 99.54 (C-6), 94.51 (C-8), 76.39 (C-3"), 77.48 (C-5"), 72.90 (C-2"), $73.80\left(\mathrm{C}-4^{\prime \prime}\right)$.

HPLC quantitative analysis - HPLC was performed on a Reverse phase JASCO HPLC system (Tokyo, Japan) equipped with a PU-1580 Intelligent HPLC pump, an LG1580-04 quaternary gradient unit, a UV-1575 intelligent UV/Vis detector, a PG-1580-54 4-line degasser, and a
CO-1560 intelligent column thermostat. Using a BORWIN chromatographic system (Le Fontanil, France), HPLC data were analyzed. Chromatography was accomplished on a Phenomenex $\mathrm{C}_{18}$ reverse phase column (Phenomenex, $4.6 \times 250 \mathrm{~mm}, 5 \mu \mathrm{m})$ at $30^{\circ} \mathrm{C}$ and monitored at $350 \mathrm{~nm}$. An isocratic solvent system consisting of $0.2 \%$ phosphoric acid and $\mathrm{MeOH}$ at ratio of 55:45 was used for $80 \mathrm{~min}$ at the flow rate of $0.5 \mathrm{ml} / \mathrm{min}$. For preparation of stock solutions, extracts and seven flavonoids were dissolved in $100 \% \mathrm{MeOH}$ at concentrations of $2 \mathrm{mg} / \mathrm{mL}$ and diluted to $1,0.5$, and $0.25 \mu \mathrm{g} / \mathrm{mL}$, respectively. After filtration through a centrifugal filter device $(0.45 \mu \mathrm{m}$, Millipore Co., Bedford, MA, USA), $10 \mu \mathrm{L}$ of each sample was injected. Calibration curves of all seven compounds were drawn with three standards at concentrations ranging from 62.5 to $500 \mu \mathrm{g} / \mathrm{mL}$. Regression equations were calculated in the form of $y=a x+b$, where $y$ and $x$ corresponded to peak area and concentration, respectively. Relative quantities of seven flavonoids in the $\mathrm{MeOH}$ extract, EtOAc fraction, and $n-\mathrm{BuOH}$ fraction $(\mathrm{mg} / \mathrm{g}$ of the extract) were calculated from each equation.

Assay for BACE1 inhibition-BACE1 inhibitory assay was carried out according to the supplied manual with selected modifications. Briefly, mixtures of $10 \mu \mathrm{L}$ of assay buffer $(50 \mathrm{mM}$ sodium acetate, $\mathrm{pH} 4.5), 10 \mu \mathrm{L}$ of BACE1 $(1.0 \mathrm{U} / \mathrm{mL})$, and $10 \mu \mathrm{L}$ of the substrate $(750 \mathrm{nM}$ Rh-EVNLDAEFK-Quencher in $50 \mathrm{mM}$, ammonium bicarbonate), and $10 \mu \mathrm{L}$ of samples (final concentration, 250 $\mu \mathrm{g} / \mathrm{mL}$ for the extracts/fractions) were incubated at $25^{\circ} \mathrm{C}$ for $60 \mathrm{~min}$ in the dark. The proteolysis of two fluorophores (Rh-EVNLDAEFK-Quencher) by BACE1 was observed by exciting the mixtures at $545 \mathrm{~nm}$ and collecting the emitted light at $585 \mathrm{~nm}$. Fluorescence was recorded with a microplate spectrofluorometer (Molecular Devices, Sunnyvale, CA, USA). The BACE1 inhibitory activity was expressed in terms of the $\mathrm{IC}_{50}$ value $(\mu \mathrm{g} / \mathrm{mL})$. Quercetin was used as a positive control.

\section{Result and Discussion}

Initially, the anti-AD potential of $\mathrm{MeOH}$ extract of $C$. maackii showed dose-dependent inhibition of BACE1 $(16.50,43.45$, and $59.48 \%$ inhibition at 20,50 , and 100 $\mu \mathrm{g} / \mathrm{mL}$, respectively). Since its $\mathrm{MeOH}$ extract showed modest inhibition, it was further portioned into different fractions. BACE1 inhibitory potentials of different fractions were then determined. Results are summarized in Table 1 and Fig. 2. $\mathrm{CH}_{2} \mathrm{Cl}_{2}(86.58 \%$ at $50 \mu \mathrm{g} / \mathrm{mL})$, EtOAc $(65.45 \%$ at $25 \mu \mathrm{g} / \mathrm{mL})$, and $n-\mathrm{BuOH}(59.63 \%$ at $100 \mu \mathrm{g} / \mathrm{mL})$ fractions showed dose-dependent inhibition against BACE1, 


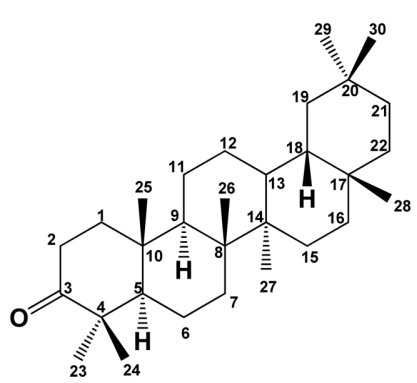

1<smiles>[R]Oc1ccc(CC2(O)C(=O)OC[C@@H]2Cc2ccc(OC)c(OC)c2)cc1OC</smiles>

5: $\mathrm{R}=\mathrm{Glu}$<smiles>[R20]c1cc(O)c2c(=O)cc(-c3ccc(O)c([R20])c3)oc2c1</smiles>

$$
\begin{aligned}
& \text { 8: } R_{1}=R_{2}=R_{3}=H \\
& \text { 9: } R_{1}=O H, R_{2}=R_{3}=H \\
& \text { 10: } R_{1}=R_{3}=H, R_{2}=G l u \\
& \text { 11: } R_{1}=O H, R_{2}=G l u, R_{3}=H \\
& \text { 12: } R_{1}=R_{2}=H, R_{3}=G \ln \\
& \text { 13: } R_{1}=O H, R_{2}=H, R_{3}=G \ln \\
& \text { 14: } R_{1}=R_{2}=H, R_{3}=G \ln M
\end{aligned}
$$

Fig. 1. Structures of the isolated compounds from Cirsium maackii. with $\mathrm{IC}_{50}$ values of $22.98 \pm 1.45,8.65 \pm 0.63$, and $72.47 \pm$ $3.04 \mu \mathrm{g} / \mathrm{mL}$, respectively, while $\mathrm{IC}_{50}$ value of quercetin, the standard, was $4.51 \pm 3.63 \mu \mathrm{g} / \mathrm{mL}$. Its $\mathrm{H}_{2} \mathrm{O}$ fraction did not show BACE1 inhibition at tested concentration (4 $100 \mu \mathrm{g} / \mathrm{mL}$ ), indicating the absence of BACE1 inhibitory potential. The inhibition order was EtOAc $>\mathrm{CH}_{2} \mathrm{Cl}_{2}>n$ $\mathrm{BuOH}$, demonstrating that EtOAc and $\mathrm{CH}_{2} \mathrm{Cl}_{2}$ fractions were remarkable BACE1 inhibitors.

Isolated compounds including $\beta$-amyrenone $(\mathbf{1}),{ }^{23}$ lupeol acetate (2), ${ }^{24}$ lupeol (3), lupenone (4), $\beta$-sitosterol
(6), $\beta$-sitosterol glucoside (7), apigenin (8), luteolin (9), tracheloside $(\mathbf{5}),{ }^{25}$ apigenin 7 -O-glucuronide methyl ester (14) ${ }^{26}$ apigenin 5 - $O$-glucoside $(\mathbf{1 0}),{ }^{27}$ luteolin 5 - $O$ glucoside (11), apigenin 7-glucuronide (12) ${ }^{28}$ and luteolin 7-O-glucuronide $(\mathbf{1 3})^{28}$ were identified via ${ }^{1} \mathrm{H}$ and ${ }^{13} \mathrm{C}$ NMR analysis as well as thin layer chromatographic analysis with standard. Data were compared with reported data. Structures of compounds are shown in Fig. 1.

In order to determine the part-specific preponderance of all the isolated flavonoids, HPLC quantitative analysis 
Table 1. BACE1 inhibitory activities of different fractions of Cirsium maackii flowers

\begin{tabular}{|c|c|c|c|}
\hline Fractions & $\mathrm{IC}_{50}$ value $(\mu \mathrm{g} / \mathrm{mL})^{\mathrm{a}}$ & Concentration $(\mu \mathrm{g} / \mathrm{mL})$ & Inhibition \% \\
\hline & & 5 & $24.40 \pm 6.49$ \\
\hline \multirow[t]{3}{*}{$\mathrm{CH}_{2} \mathrm{Cl}_{2}$} & $22.98 \pm 1.45$ & 10 & $33.54 \pm 1.92$ \\
\hline & & 50 & $86.58 \pm 1.47$ \\
\hline & & 2 & $26.98 \pm 2.85$ \\
\hline \multirow[t]{3}{*}{ EtOAc } & $8.65 \pm 0.63$ & 10 & $54.62 \pm 2.05$ \\
\hline & & 25 & $65.45 \pm 0.74$ \\
\hline & & 4 & $20.68 \pm 2.32$ \\
\hline \multirow[t]{3}{*}{$n-\mathrm{BuOH}$} & $72.47 \pm 3.04$ & 20 & $34.56 \pm 0.29$ \\
\hline & & 100 & $59.63 \pm 1.39$ \\
\hline & & 4 & $21.34 \pm 1.78$ \\
\hline \multirow[t]{3}{*}{$\mathrm{H}_{2} \mathrm{O}$} & $>100$ & 20 & $25.49 \pm 2.50$ \\
\hline & & 100 & $31.38 \pm 5.16$ \\
\hline & & 0.6 & $33.06 \pm 1.67$ \\
\hline \multirow[t]{2}{*}{ Quercetin $^{\mathrm{b}}$} & $4.51 \pm 3.63$ & 3 & $49.06 \pm 0.91$ \\
\hline & & 6 & $55.82 \pm 2.52$ \\
\hline
\end{tabular}

${ }^{\mathrm{a}}$ The $50 \%$ inhibition concentrations $\left(\mathrm{IC}_{50}, \mu \mathrm{g} / \mathrm{mL}\right)$ are expressed as mean $\pm \mathrm{SD}$ of triplicates.

bositive control.

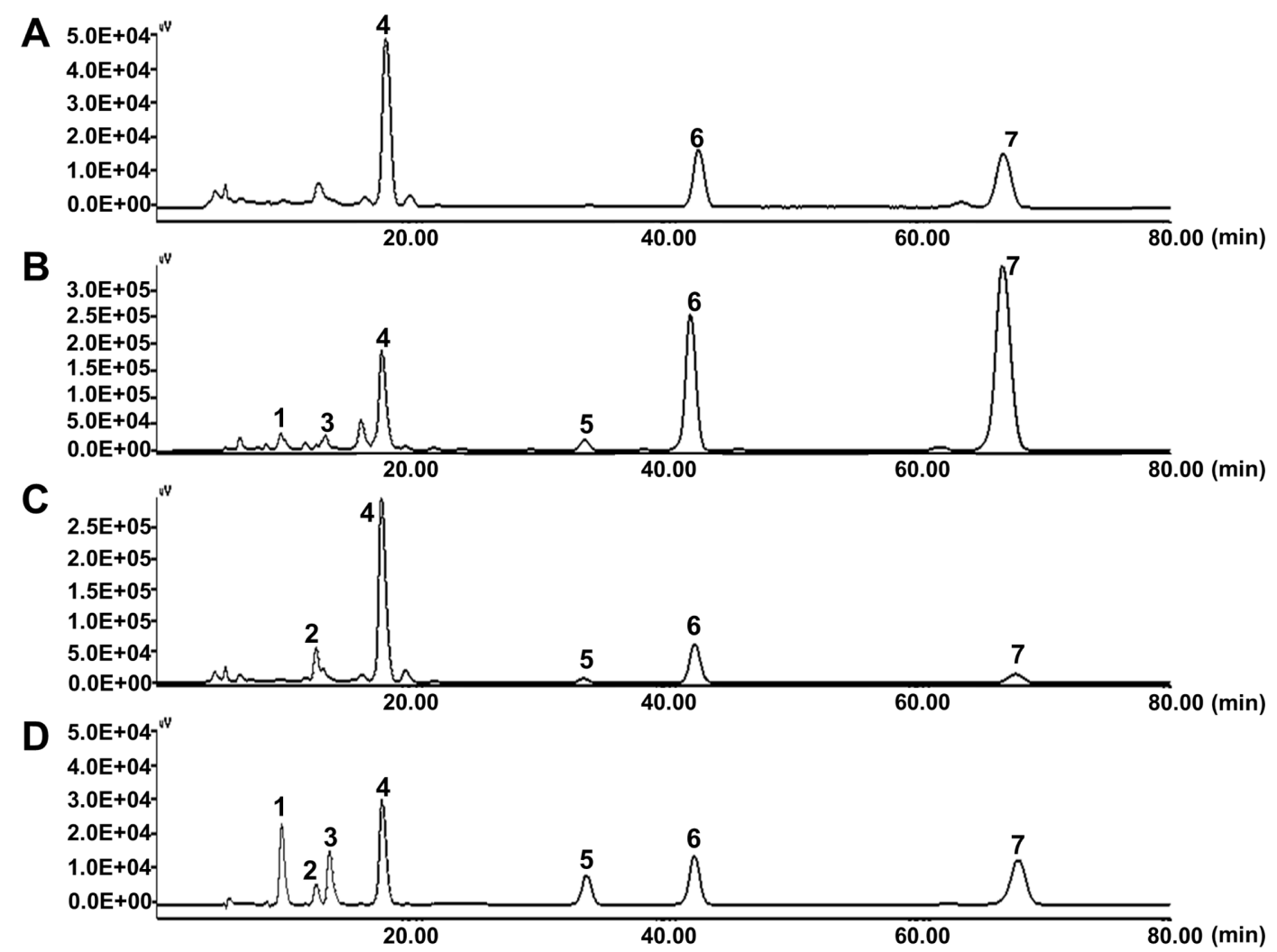

Fig. 2. HPLC chromatograms of $\mathrm{MeOH}$ extract and different fractions of Cirsium maackii flower. (A) MeOH extract, (B) EtOAc fraction, (C) $n$-BuOH fraction along with (D) standard compounds: peak 1 (RT: 9.84 min, compound 11), peak 2 (RT: 12.57 min, compound 13), peak 3 (RT: $13.60 \mathrm{~min}$, compound 10), peak 4 (RT: $17.74 \mathrm{~min}$, compound 12), peak 5 (RT: 33.87 min, compound 14), peak 6 (RT: 42.38 min, compound 9), peak 7 (RT: 67.93 min, compound 8).

of $\mathrm{MeOH}$ extract, EtOAc fraction, and $n-\mathrm{BuOH}$ of $C$. maackii flowers was performed. Chromatograms, calibra- tion curve, and quantification data are shown in Fig. 2 and Tables 2 and 3, respectively. The $\mathrm{MeOH}$ extract had the 
Table 2. HPLC calibration curve data of compounds 8 - 14 .

\begin{tabular}{cccc}
\hline \hline Compounds & Retention time (min) & Linear Regression Equation & $\mathrm{R}^{2}$ \\
\hline $\mathbf{8}$ & 67.93 & $\mathrm{y}=9953690.54 \mathrm{x}-5317178.63$ & 0.999 \\
$\mathbf{9}$ & 42.38 & $\mathrm{y}=9784471.21 \mathrm{x}-5398920.96$ & 0.999 \\
$\mathbf{1 0}$ & 13.60 & $\mathrm{y}=1404260.46 \mathrm{x}+687709.38$ & 0.998 \\
$\mathbf{1 1}$ & 9.84 & $\mathrm{y}=2227168.42 \mathrm{x}+504059.63$ & 0.996 \\
$\mathbf{1 2}$ & 17.74 & $\mathrm{y}=4546174.33 \mathrm{x}+405951.05$ & 0.998 \\
$\mathbf{1 3}$ & 12.57 & $\mathrm{y}=632193.91 \mathrm{x}+549692.85$ & 0.995 \\
$\mathbf{1 4}$ & 33.87 & $\mathrm{y}=2304160.45 \mathrm{x}-303419.83$ & 0.998 \\
\hline
\end{tabular}

Table 3. Relative amounts of isolated compounds in extracts and fractions of Cirsium maackii flowers

\begin{tabular}{lrrrcrrr}
\hline \multicolumn{1}{c}{$\begin{array}{c}\text { Extract/ } \\
\text { Fraction }\end{array}$} & \multicolumn{7}{c}{ Amount of compounds in the extract/fraction (mg/g) $)^{\mathrm{a}}$} \\
\cline { 2 - 8 } & Compound $\mathbf{8}$ & Compound 9 & \multicolumn{1}{c}{ Compound 10 } & Compound 11 & Compound 12 & Compound 13 & Compound 14 \\
\hline MeOH extract & $33.07 \pm 0.07$ & $31.44 \pm 0.17$ & ND & ND & $16.89 \pm 0.33$ & ND & $6.66 \pm 0.01$ \\
EtOAc fraction & $161.01 \pm 1.78$ & $96.93 \pm 0.34$ & $8.26 \pm 0.07$ & $16.80 \pm 1.01$ & $73.38 \pm 0.06$ & ND & $27.03 \pm 1.94$ \\
$n$-BuOH fraction & $32.18 \pm 0.33$ & $44.31 \pm 0.32$ & $23.39 \pm 0.84$ & ND & $105.94 \pm 0.36$ & $60.62 \pm 0.83$ & $13.42 \pm 0.10$ \\
\hline
\end{tabular}

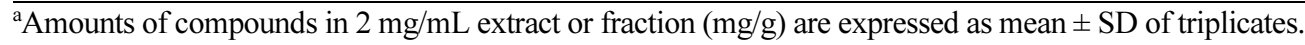

ND Not detected.

highest amount of apigenin (8) and luteolin (9) followed by apigenin 7-O-glucuronide (12). The calibration curve of for all seven compounds luteolin 5-O-glucoside (11), apigenin 5-O-glucoside (10), apigenin 7-O-glucuronide (12), apigenin 7- $O$-glucuronide methyl ester (14), luteolin (9), and apigenin (8) were drawn at 0.5, 0.25 and 0.125 $\mathrm{mg} / \mathrm{mL}$ as shown in Table 2. Using linear regression equation obtained from the calibration curve, the number of individual compounds in $2 \mathrm{mg} / \mathrm{mL}$ extract and fractions were calculated. The relative amount of apigenin 7-Oglucuronide (12), apigenin 7-O-glucuronide methyl ester (14), luteolin (9), and apigenin (8) in the $\mathrm{MeOH}$ extracts from C. maackii flowers were $16.89 \pm 0.33 \mathrm{mg} / \mathrm{g}, 6.66 \pm$ $0.01 \mathrm{mg} / \mathrm{g}, \quad 31.44 \pm 0.17 \mathrm{mg} / \mathrm{g}$, and $33.07 \pm 0.07 \mathrm{mg} / \mathrm{g}$, respectively. The yield of the EtOAc fraction for luteolin 5-O-glucoside (11), apigenin 5-O-glucoside (10), apigenin 7-O-glucuronide (12), apigenin 7-O-glucuronide methyl ester (14), luteolin (9), and apigenin (8) were 16.80 \pm 1.01 $\mathrm{mg} / \mathrm{g}, 8.26 \pm 0.07 \mathrm{mg} / \mathrm{g}, 73.38 \pm 0.06 \mathrm{mg} / \mathrm{g}, 27.03 \pm 1.94$ $\mathrm{mg} / \mathrm{g}, 96.93 \pm 0.34 \mathrm{mg} / \mathrm{g}$, and $161.01 \pm 1.78 \mathrm{mg} / \mathrm{g}$ indicating that apigenin (8) is abundant in EtOAc fraction. The contents of luteolin 7-O-glucuronide (13), apigenin 5-Oglucoside (10), apigenin 7-O-glucuronide (12), apigenin 7-O-glucuronide methyl ester (14), luteolin (9), and apigenin (8) in the $n$-BuOH fraction of flowers were $60.62 \pm 0.83$, $22.39 \pm 0.84,105.94 \pm 0.36,13.42 \pm 0.10,44.31 \pm 0.32$, and $32.18 \pm 0.33 \mathrm{mg} / \mathrm{g}$ respectively, showing that the presence of apigenin 7-O-glucuronide (12) is abundant in $n$-BuOH fraction.

$\mathrm{AD}$ is one of detrimental neurodegenerative diseases in this aging society. It demands instant attention. Since some plant flowers have been reported to possess effect against inhibition of BACE1, ${ }^{29}$ this study was undertaken to evaluate the anti-AD potential of $C$. maackii flower together with quantification of its major compounds. Our results demonstrated the potential of $C$. maackii flower $\mathrm{MeOH}$ extract and its $\mathrm{CH}_{2} \mathrm{Cl}_{2}$ and EtOAc fractions in alleviating $\mathrm{AD}$ by inhibiting BACE1. In urge to identify the key compounds in our extract and fraction, we conducted HPLC and column chromatography analysis which revealed the presence of significant amounts of BACE1 inhibiting compounds lupeol (3) and luteolin (9) in $\mathrm{CH}_{2} \mathrm{Cl}_{2}$ and EtOAc fraction, respectively.

Our results showed that the EtOAc fraction had the highest BACE1 inhibiting activity. HPLC analysis revealed that two flavonoids, apigenin (8) and luteolin (9), had the highest amounts in the EtOAc fraction. These two compounds in the EtOAc fraction might have the highest contribution to the anti-AD property. As evidenced in our previous work, ${ }^{30}$ luteolin (9) has incredible BACE1 inhibiting effect, with $\mathrm{IC}_{50}$ of $1.95 \pm 0.02 \mu \mathrm{M}$, which is higher even than the positive control, quercetin $(3.17 \pm$ $0.12 \mu \mathrm{M})$. Similarly, in the cerebral cortex of a mouse model, luteolin has been reported to exert neurovascular protection against $A \beta_{25-35}$-induced amnesia, improve spatial learning and memory capabilities, modulate microvascular function, increase regional cerebral blood flow values, combat reactive oxygen species, improve cholinergic neuronal system, and increase brain-derived neurotrophic factor level and its receptor tyrosine kinase B expression. ${ }^{31}$ Wang et al. have also highlighted the potential effect of luteolin (9) on learning defects and 
hippocampal structure in $\mathrm{AD} .{ }^{32}$ Moreover, apigenin (8) possesses scavenging effects against superoxide anion. It can improve anti-oxidative enzyme activity of superoxide dismutase and glutathione peroxidase and restore the neurotrophic ERK/CREB/BDNF pathway in the cerebral cortex, suggesting its ability to ameliorate $\mathrm{AD}$-associated learning and memory impairment by relieving $A \beta$ burden. ${ }^{33}$ Since contents of luteolin $(9)$ and apigenin $(\mathbf{8})$ in EtOAc fraction were the highest, apigenin (8) might be able to synergize the BACE1 inhibitory activity of luteolin in the EtOAc fraction of $C$. maackii flower.

$\mathrm{CH}_{2} \mathrm{Cl}_{2}$ fraction also showed a notable degree of inhibition against BACE1. Column chromatography of $C$. maackii flower revealed that this fraction was rich in lupeol (3) and lupeol acetate (2). Our previous report has revealed that lupeol (3) could effectively inhibit BACE1 enzyme, with $\mathrm{IC}_{50}$ of $5.12 \pm 0.30 \mu \mathrm{M} \cdot{ }^{34}$ In addition, it has been reported that lupeol (3) can restore $A \beta_{1-42}$ induced behavioral and biochemical abnormalities in rat brain. ${ }^{35}$ Thus, the activity of $\mathrm{CH}_{2} \mathrm{Cl}_{2}$ fraction of $C$. maackii flower against BACE1 might be correlated with the inhibitory activity of lupeol (3).

Emerging evidences suggest a close association between oxidative stress and neurotoxicity of amyloid fibrils. ${ }^{36}$ Jung et al. have shown convincing activity of C. maackii flower against oxidative mediators including 2,2-diphenyl-1picrylhydrazyl (DPPH), peroxynitrite $\left(\mathrm{ONOO}^{-}\right)$, reactive oxygen species (ROS), and rat lens aldose reductase (RLAR) inhibition, thereby suggesting its anti-oxidative potential. ${ }^{8}$ Furthermore, its EtOAc fraction was highly active in all tested parameters while $\mathrm{CH}_{2} \mathrm{Cl}_{2}$ fraction was active against $\mathrm{ONOO}^{-}$only. ${ }^{8}$ Since luteolin (9) has been reported to possess potent RLAR and DPPH inhibiting activity ${ }^{37}$ the anti-BACE1 activity of EtOAc fraction of C. maackii flower might be associated with the antioxidative potential of luteolin (9) which had the highest content in the EtOAc fraction.

Conversely, $n-\mathrm{BuOH}$ fraction of C. maackii flower had the modest activity compared to the two other nonpolar fractions. HPLC analysis showed that two glucuronides (apigenin 7-O- $\beta$-D-glucuronide (12) and luteolin 7-O- $\beta$-Dglucuronide (13)) had high contents. Iakovleva et al. have reported that luteolin, but not luteolin 7-O- $\beta$-D-glucoside (13), can attenuate cytotoxic response of cultured neuronal cells to transthyretin, a plasma protein with amyloidogenic property, and rescue the phenotype of a Drosophila melanogaster model. ${ }^{38}$ The presence of glucoronidated forms of apigenin (8) and luteolin (9) in a large amount in the $n$ - $\mathrm{BuOH}$ fraction might have contributed to the lower activity of this fraction.
To the best of our knowledge, this is the first study to isolate secondary metabolites from $C$. maackii flowers through column chromatography. This is the first report on the isolation of $\beta$-amyrenone (1), lupeol acetate (2), lupeol (3), lupenone (4), $\beta$-sitosterol (6), and $\beta$-sitosterol glucoside (7) as non-polar constituents of $C$. maackii flower. Previously, Jung et al. have performed HPLC analysis of $C$. maackii flower and showed the presence of apigenin (8), luteolin (9), apigenin 7-O-glucuronide (12), and luteolin 5-O-glucoside (11) in its $\mathrm{MeOH}$ extract. ${ }^{8} \mathrm{In}$ the present study, we were able to isolate these compounds together with apigenin 7-O-glucuronide methyl ester (14), tracheloside (5), apigenin 5-O-glucoside (10), and luteolin 7-O-glucuronide (13) from C. maackii flowers. Although the presence of a rare flavonoid, apigenin 5-Oglucoside (10), has been long described by Iwashina et al. in Japanese Cirsium species by principal component and UV spectra analysis, this is the first chromatographic separation and quantification of this compound from $C$. maackii. ${ }^{39}$ Additionally, using HPLC, we were able to quantify amounts of compounds in the $\mathrm{MeOH}$ extract as well as in EtOAc and $n-\mathrm{BuOH}$ fractions.

Flowering part of a plant is the most underexploited part compared to other plant parts such as leaves, root, and stem. During cultivation of C. maackii plant, the flowering part might be used as a source of important bioactive molecules or to be developed as a nutraceutical. Flowers of $C$. maackii are available plenteously during the flowering season at low cost or free of cost, suggesting that the thistle flower not only has aesthetic property, but also therapeutic potential to ameliorate $\mathrm{AD}$.

In conclusion, the presence of major reference compounds, lupeol (3) and luteolin (9), from C. maackii flowers have potential in the treatment of $\mathrm{AD}$ as the fraction containing them exhibited anti-BACE1 activity. Our current findings suggest that flowers of $C$. maackii might have therapeutic potential for treating patients with $\mathrm{AD}$. Since these results were based on in vitro tests, further experiments are needed to verify considering the mechanism of compounds isolated from C. maackii flowers.

\section{Acknowledgments}

This research was supported by Research Grant of Pukyong National University (Granted in 2019).

\section{References}

(1) Reitz, C. Int. J. Alzheimers Dis. 2012, 2012, 369808. 
(2) Singh, S. K.; Srivastav, S.; Yadav, A. K.; Srikrishna, S.; Perry, G. Oxid. Med. Cell. Longev. 2016, 2016, 7361613.

(3) Fedele, E.; Rivera, D.; Marengo, B.; Pronzato, M. A.; Ricciarelli, R. Mech. Ageing Dev. 2015, 152, 1-4.

(4) Kumar, A.; Singh, A.; Ekavali. Pharmacol. Rep. 2015, 67, 195-203.

(5) Das, B.; Yan, R. CNS Drugs 2019, 33, 251-263.

(6) Yan, R.; Vassar, R. Lancet Neurol. 2014, 13, 319-329.

(7) Shin, M. S.; Park, J. Y.; Lee, J.; Yoo, H. H.; Hahm, D. H.; Lee, S. C.; Lee, S.; Hwang, G. S.; Jung, K.; Kang, K. S. Bioorg. Med. Chem. Lett. 2017, 27, 3076-3080.

(8) Jung, H. A.; Kim, Y. S.; Choi, J. S. Food Chem. Toxicol. 2009, 47, 2790-2797.

(9) Nazaruk, J.; Jakoniuk, P. J. Ethnopharmacol. 2005, 102, 208-212.

(10) Sahli, R.; Rivière, C.; Dufloer, C.; Beaufay, C.; Neut, C.; Bero, J.; Hennebelle, T.; Roumy, V.; Ksouri, R.; Quetin-Leclercq, J.; Sahpaz, S. Evid. Based Complement. Alternat. Med. 2017, 2017, 7247016.

(11) Yin, J.; Heo, S. I.; Wang, M. H. Nutr. Res. Pract. 2008, 2, 247-251.

(12) Nazaruk, J.; Wajs-Bonikowska, A.; Bonikowski, R. Chem. Nat. Compd. 2012, 48, 8-10.

(13) Kim, E. Y.; Jho, H. K.; Kim, D. I.; Rhyu, M. R. J. Ethnopharmacol. 2008, 116, 223-227.

(14) Liu, S.; Zhang, J.; Li, D.; Liu, W.; Luo, X.; Zhang, R.; Li, L.; Zhao, J. Nat. Prod. Res. 2007, 21, 915-922.

(15) Ma, Q.; Guo, Y.; Luo, B.; Liu, W.; Wei, R.; Yang, C.; Ding, C.; Xu, X.; He, M. Nat. Prod. Res. 2016, 30, 1824-1829.

(16) Jung, H. A.; Park, J. J.; Min, B. S.; Jung, H. J.; Islam, M. N.; Choi, J. S. Asian Pac. J. Trop. Med. 2015, 8, 1-5.

(17) Könye, R.; Tóth, G.; Sólyomváry, A.; Mervai, Z.; Zürn, M.; Baghy, K.; Kovalszky, I.; Horváth, P.; Molnár-Peri, I.; Noszál, B.; Béni, S.; Boldizsár, I. Fitoterapia 2018, 127, 413-419.

(18) Lai, W. C.; Wu, Y. C.; Dankó, B.; Cheng, Y. B.; Hsieh, T. J.; Hsieh, C. T.; Tsai, Y. C.; El-Shazly, M.; Martins, A.; Hohmann, J.; Hunyadi, A.; Chang, F. R. J. Nat. Prod. 2014, 77, 1624-1631.

(19) Chung, M. J.; Lee, S.; Park, Y. I.; Lee, J.; Kwon, K. H. Life Sci. 2016, 148, 173-182.

(20) Luan, N.; Wei, W. D.; Wang, A.; Wu, X. L.; Qi, Y.; Li, J. J.; Zheng, J. Q.; Shang, X. Y. J. Asian Nat. Prod. Res. 2016, 18, 1015-1023.

(21) Walesiuk, A.; Nazaruk, J.; Braszko, J. J. J. Ethnopharmacol. 2010, 129, 261-266.

(22) Kim, S. J.; Kim, G. H. J. Food Sci. Nutr. 2003, 8, 330-335.

(23) Quintao, N. L.; Rocha, L. W.; Silva, G. F.; Reichert, S., Claudino, V. D.; Lucinda-Silva, R. M.; Malheiros, A.; De Souza, M. M.; Filho, V.
C.; Bresolin, T. M.; Machado, M.; Wagner, T. M.; Silva, C. M. BioMed. Res. Int. 2014, 2014, 636839

(24) Fernández-Martínez, E.; Jiménez-Santana, M.; Centeno-Álvarez, M.; Torres-Valencia, J. M.; Shibayama, M.; Cariño-Cortés, R. Pharmacogn. Mag. 2017, 13, S860-S867.

(25) Johansen, K. T.; Wubshet, S. G.; Nyberg, N. T.; Jaroszewski, J. W. J. Nat. Prod. 2011, 74, 2454-2461.

(26) Mostafa, N. M.; Eldahshan, O. A.; El-Beshbishy, H. A.; Singab, A. N. Med. Aromat. Plant (Los Angles) 2017, 6, 1000297.

(27) Veit, M.; Geiger, H.; Czygan, F. C.; Markham, K. R. Phytochemistry 1990, 29, 2555-2560.

(28) Ozgen, U.; Sevindik, H.; Kazaz, C.; Yigit, D.; Kandemir, A.; Secen, H.; Calis, I. Molecules 2010, 15, 2593-2599.

(29) Liu, Q.; Fang, J.; Wang, P.; Du, Z.; Li, Y.; Wang, S.; Ding, K. Int. J. Biol. Macromol. 2018, 107, 112-120.

(30) Choi, J. S.; Islam, M. N.; Ali, M. Y.; Kim, Y. M.; Park, H. J.; Sohn, H. S.; Jung, H. A. Arch. Pharm. Res. 2014, 37, 1354-1363.

(31) Liu, R.; Gao, M.; Qiang, G. F.; Zhang, T. T.; Lan, X.; Ying, J.; Du, G. H. Neuroscience 2009, 162, 1232-1243.

(32) Wang, H.; Wang, H.; Cheng, H.; Che, Z. Mol. Med. Rep. 2016, 13, 4215-4220.

(33) Zhao, L.; Wang, J. L.; Liu, R.; Li, X. X.; Li, J. F.; Zhang, L. Molecules 2013, 18, 9949-9965.

(34) Koirala, P.; Seong, S. H.; Jung, H. A.; Choi, J. S. Asian Pac. J. Trop. Med. 2017, 10, 1117-1122.

(35) Kaundal, M.; Akhtar, M.; Deshmukh, R. J. Neurol. Neurosci. 2017, $8,195$.

(36) Chakraborty, S.; Basu, S. Int. J. Biol. Macromol. 2017, 103, 733743.

(37) Hwang, S. H.; Wang, Z.; Guillen Quispe, Y. N.; Lim, S. S.; Yu, J. M. J. Diabetes Res. 2018, 2018, 3276162.

(38) Iakovleva, I.; Begum, A.; Pokrzywa, M.; Walfridsson, M.; SauerEriksson, A. E.; Olofsson, A. PLoS One 2015, 10, e0128222.

(39) Iwashina T.; Ito, T.; Ootani, S. Ann. Tsukuba Bot. Gard. 1989, 8, 15-19.

Received July 3, 2019

Revised October 15, 2019 Accepted October 16, 2019 